proposed as a therapeutic alternative in these patients. Janus Kinase (JAK) inhibitors in combination to the antiviral Remdesivir have shown evidence of reducing recovery time and accelerating improvement in clinical status among patients with COVID-19, notably among those receiving high-flow oxygen or noninvasive ventilation ${ }^{1}$.

Objectives: To describe the outcomes associated with the use of Tocilizumab (TCZ) and Baricitinib (Bari) in patients hospitalized for COVID-19.

Methods: Descriptive study nested in a cohort. Patients from the General Hospital of Cuernavaca who were hospitalized due to COVID-19 associated pneumonia and/or respiratory failure requiring supplemental oxygen or invasive/ non-invasive assisted mechanical ventilation (AMV) were analyzed. All patients had a positive RT-PCR upon hospital admission, sampled under standardized conditions. The clinical and demographic data of the patients upon hospitalization were obtained from the instrument on a specific platform (SISVER) used on a national basis. Severe disease was considered as the need for any type of AMV. TCZ was used at $400-600 \mathrm{mg}$ in two infusions (baseline and at 72 hours), Bari at $4 \mathrm{mg}$ daily for 14 days. The outcomes were the need for AMV, death, or discharge. A $p \leq 0.05$ was considered statistically significant.

Results: Data from 404 SARS-CoV2 positive patients were analyzed. 269 $(65.9 \%)$ were men. The mean age of the patients was 57.5 years (18-94; SD 15.3). $59.6 \%$ of the patients had one or more comorbidities (Diabetes and Hypertension in most cases [31.8\%]). $22.8 \%$ of the patients had a history of current or previous smoking. Mean hospital stay was 7.4 days (1-36; SD 5.9). 15.9\% of the patients required invasive or non-invasive AMV. 11 patients were treated with TCZ and 30 patients received treatment with Baricitinib. 5 of the 11 patients with TCZ required AMV (all but one were intubated), but only 6 of the $30(20 \%)$ with Bari required AMV (all except one received CPAP). The use of Baricitinib was correlated with a reduction in the use of AMV ( $p$ 0.01). 6 patients with TCZ were discharged due to improvement and 5 died. In the case of Bari, 19 improved and were discharged and 11 died, significantly correlating with lower mortality ( $p$ 0.05). The use TCZ was not associated with a reduced hospitalization (50\% remained hospitalized for more than 10 days) but patients receiving Baricitinib had significantly shorter hospital stays $(86.6 \%$ had a hospital stay of less than 10 days) than those receiving TCZ $(p<0.0001)$

Conclusion: The patients that were treated with Baricitinib and required AMV most often improved with non-invasive CPAP while the majority of the patients under AMV receiving TCZ were treated with an invasive mode, although this was due in part to the fact that TCZ can be employed intravenously and is more practical for intubated patients in whom oral drug administration is limited. Baricitinib was significantly associated with a better outcome (hospital discharge due to improvement) and a significantly shorter hospital stay.

REFERENCES:

[1] Kalil, A.C.; Patterson, T.F.; Mehta, A.K.; Tomashek, K.M.; Wolfe, C.R.; Ghazaryan, V.; Marconi, V.C.; Ruiz-Palacios, G.M.; Hsieh, L.; Kline, S.; et al. Baricitinib plus Remdesivir for Hospitalized Adults with Covid-19. N. Engl. J. Med. 2020.

Acknowledgements: Thanks to B. Flores and M. Hernandez for their help with data collection.

Disclosure of Interests: Daniel Xavier Xibille Friedmann Speakers bureau: Lilly, Abbvie, Paid instructor for: Lilly, Abbvie, Consultant of: Pfizer, Lilly, Sandra Miriam Carrillo Vazquez Speakers bureau: Roche, Novartis, Paid instructor for: Roche, Janssen, Consultant of: Roche, Janssen

DOI: 10.1136/annrheumdis-2021-eular.3122

\section{POS1234 \\ IMPACT OF THE CHANGE IN ADMINISTRATION ROUTE OF TOCILIZUMAB AND ABATACEPT, DUE TO THE COVID-19 LOCKDOWN ON DISEASE ACTIVITY IN PATIENTS WITH RHEUMATOID ARTHRITIS}

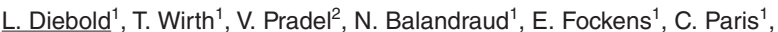
S. Trijau', P. Lafforgue ${ }^{1}$, T. Pham ${ }^{1} .{ }^{1}$ APHM, Rheumatology Department, Marseille, France; ${ }^{2}$ APHM, Public Health Department, Marseille, France

Background: Among therapeutics used to treat rheumatoid arthritis (RA), Tocilizumab (TCZ) and Abatacept (ABA) are both biologic agents that can be delivered subcutaneously (SC) or intravenously (IV). During the first COVID-19 lockdown in France, all patients treated with IV TCZ or IV ABA were offered the option to switch to SC administration.

Objectives: The primary aim was to assess the impact of changing the route of administration on the disease activity. The second aim was to assess whether the return to IV route at the patient's request was associated with disease activity variation, flares, anxiety, depression and low physical activity during the lockdown.

Methods: We conducted a prospective monocentric observational study. Eligibility criteria: Adult $\geq 18$ years old, RA treated with IV TCZ or IV ABA with a stable dose $\geq 3$ months, change in administration route (from IV to SC) between March 16,2020 , and April 17, 2020. The following data were collected at baseline and 6 months later (M6): demographics, RA characteristics, treatment, history of previous SC treatment, disease activity (DAS28), self-administered questionnaires on flares, RA life repercussions, physical activity, anxiety and depression (FLARE, RAID, Ricci \&Gagnon, HAD).

The primary outcome was the proportion of patients with a DAS28 variation $>1.2$ at M6. Analyses: Chi2-test for quantitative variables and Mann-Whitney test for qualitative variables. Factors associated with return to IV route identification was performed with univariate and multivariate analysis.

Results: Among the 84 patients who were offered to switch their treatmen route of administration, 13 refused to change their treatment. Among the 71 who switched (48 TCZ, $23 \mathrm{ABA}$ ), 58 had a M6 follow-up visit (13 lost of follow-up) and DAS28 was available for 49 patients at M6. Main baseline characteristics: female $81 \%$, mean age 62.7 , mean disease duration: 16.0 , ACPA positive: $72.4 \%$, mean DAS28: 2.01, previously treated with SC TCZ or ABA: $17 \%$.

At M6, the mean DAS28 variation was $0.18 \pm 0.15$. Ten $(12.2 \%)$ patients had a DAS28 worsening $>1.2$ (ABA: $5 / 17$ [29.4\%] and TCZ: $5 / 32$ [15.6\%], $p=0.152$ ) and 19 patients $(32.8 \%)$ had a DAS28 worsening $>0.6$ (ABA: $11 / 17[64.7 \%]$ and TCZ: $8 / 32[25.0 \%], p=0.007)$.

At M6, 41 patients $(77.4 \%)$ were back to IV route (26 TCZ, $15 \mathrm{ABA}$ ) at their request. The proportion of patients with a DAS28 worsening $>1.2$ and $>0.6$ in the groups return to IV versus SC maintenance were $22.5 \%, 42.5 \%$ versus $11.1 \%$ and $22.2 \%(p=0.4)$, respectively. The univariate analysis identified the following factors associated with the return to IV route: HAD depression score (12 vs 41 $\mathrm{p}=0.009)$, HAS anxiety score ( 12 vs $41, \mathrm{p}=0.047)$ and corticosteroid use $(70 \%$ vs $100 \%, p=0.021$ ), in the SC maintenance vs return to IV, respectively

Conclusion: The change of administration route of TCZ and ABA during the first COVID-19 lockdown was infrequently associated with a worsening of RA disease. However, the great majority of the patients $(77.4 \%)$ request to return to IV route, even without disease activity worsening. This nocebo effect was associated with higher anxiety and depression scores.

Disclosure of Interests: None declared

DOI: 10.1136/annrheumdis-2021-eular.3126

\section{\begin{tabular}{|l|l}
\hline POS1235 & ASSESSMENT OF RISK FACTORS FOR
\end{tabular} HOSPITALIZATION OF AUTOIMMUNE AND RHEUMATIC DISEASE PATIENTS WITH COVID-19 - A SINGLE CENTRE OBSERVATIONAL STUDY}

J. M N ${ }^{1}$, P. Dekate ${ }^{2}$, S. Vontari ${ }^{1}$, R. Dudam3. ${ }^{1}$ Hyderabad Rheumatology Center, Clinical Pharmacology, Hyderabad, India; ${ }^{2}$ Hyderabad Rheumatology Center, Rheumatology, Hyderabad, India; ${ }^{2}$ Hyderabad Rheumatology Center, Rheumatology, Hyderabad, India

Background: Most of the patients suffering from autoimmune and rheumatic diseases (AIRD) are on immunomodulator or immunosuppressive therapies and they are susceptible to infections including COVID19.

Objectives: To assess the risk factors for hospitalization with COVID-19 in patients with AIRD

Methods: A single centre retrospective observational study including patients with AIRD who had contracted COVID-19. Data was collected from all patients attending our clinic from September 2020 to January 2021. Patients with COVID19 were divided into hospitalized and non-hospitalized groups. Clinical, demographic, ongoing medications, comorbidities and post COVID flare data were collected and analysed using crosstabs-chi square and Mann-Whitney $U$ test.

Results: 520 patients with AIRD were screened over the study duration, of which 52 patients who had contracted COVID-19 were included in the study. Amongst them, 19 patients $(36.5 \%)$ required hospitalization. The common symptoms in the hospitalized patients over non-hospitalized were fever $(63.2 \%$ vs 57.6 ), fatigue ( $42.1 \%$ vs $21.2 \%$ ), headache (36.8\% vs $18.2 \%$ ), abdominal pain ( $26.3 \%$ vs $6.1 \%$ ), myalgias ( 36.8 vs $18.2 \%$ ) and dyspnea ( $31.6 \%$ vs $18.2 \%)$. The most common rheumatic conditions seen in both the groups were RA $(42.1 \%$ vs $30.3 \%$ ), SPA (26.3\% vs $36.4 \%$ ), and SLE with other CTD's (15.8\% vs $24.2 \%$ ). 6 patients (11.5\%) required ICU stay, 3 patients $(5.7 \%)$ were ventilated, and 2 (3.8\%) patients died.

Patients requiring hospitalization were aged $>50$ years $(p=0.018)$, had DM type I $(p=0.003)$, HTN $(p=0.035)$, multimorbidity $(p=0.021)$, and a higher CORAD score $(p<0.001)$. Usage of CsDMARDS, bDMARDS, TsDMARDS, corticosteroids did not show a significant difference between both the groups. Patients with $>2$ years of disease duration required hospitalization in comparison to $<2$ years of duration but this was not statistically significant.

$84.2 \%$ of hospitalized and $69.7 \%$ of non-hospitalized patients stopped anti-rheumatic treatment and disease flare was seen in $47.4 \%$ and $39.4 \%$ patients respectively.

Conclusion: The rheumatic disease and their medications did not increase the risk of COVID-19 hospitalization in this study. However, higher age, DM II, HTN and high CORAD score were found to be associated with hospitalization in AIRD patients. The findings of this study are limited by a small sample size. 
Table 1. Demographic and Rheumatic disease specific data of COVID-19 patients

\begin{tabular}{|c|c|c|c|c|c|c|c|c|c|c|c|}
\hline & & $\begin{array}{l}\text { All patients } \\
\qquad(n=52)\end{array}$ & $\begin{array}{l}\text { Hospitalized } \\
\qquad(n=19)\end{array}$ & $\begin{array}{c}\text { Non-hospitalized } \\
(n=33)\end{array}$ & p-value & & & $\begin{array}{l}\text { All patients } \\
\quad(n=52)\end{array}$ & $\begin{array}{l}\text { Hospitalized } \\
\qquad(n=19)\end{array}$ & $\begin{array}{l}\text { Non-hospitalized } \\
\qquad(\mathrm{n}=33)\end{array}$ & $\begin{array}{c}\mathrm{p}- \\
\text { value }\end{array}$ \\
\hline \multicolumn{2}{|l|}{ Age } & $45.73(10.829)$ & $50.53(10.905)$ & $42.97(9.923)$ & 0.018 & Duration & $>2$ years & $14(26.9)$ & $3(15.8)$ & $11(33.3)$ & 0.147 \\
\hline \multirow{2}{*}{\multicolumn{2}{|c|}{$\begin{array}{l}\text { - n (\%) } \\
\text { Gender (Male/Female) }\end{array}$}} & & & & & $-n(\%)$ & $>2$ years & $38(73.1)$ & $16(84.2)$ & $22(66.7)$ & \\
\hline & & $21(40.4) / 31$ & $7(36.8) / 12$ (63.2) & $14(42.4) / 19(57.6)$ & 0.462 & Corticosteroids & No steroids & $18(34.6)$ & $5(26.3)$ & 13(39.4) & 0.294 \\
\hline & & $(59.6)$ & & & & $-n(\%)$ & Low dose steroids & $33(63.5)$ & $13(68.4)$ & $20(60.6)$ & \\
\hline \multirow[t]{3}{*}{$\begin{array}{l}\text { BMI } \\
-n(\%)\end{array}$} & Normal & $27(51.9)$ & $8(42.1)$ & $19(57.6)$ & 0.528 & & $\begin{array}{c}\text { Moderate dose } \\
\text { steroids }\end{array}$ & $1(1.9)$ & $1(5.3)$ & $0(0.0)$ & \\
\hline & Pre-obese & $19(36.5)$ & $8(42.1)$ & $11(33.3)$ & & DMARDS (CS) & MTX & $25(48.1)$ & $8(42.1)$ & $17(51.5)$ & 0.358 \\
\hline & Obesity & $6(11.5)$ & $3(15.8)$ & $3(9.1)$ & & $-n(\%)$ & $\mathrm{HCQ}$ & $13(25)$ & $5(26.3)$ & $8(24.2)$ & 0.560 \\
\hline \multirow{4}{*}{$\begin{array}{l}\text { Disease } \\
-n(\%)\end{array}$} & RA & 18(34.6) & $8(42.1)$ & $10(30.3)$ & 0.287 & & LEF & $4(7.7)$ & $3(15.8)$ & $1(3)$ & 0.132 \\
\hline & SPA & $17(32.7)$ & $5(26.3)$ & $12(36.4)$ & 0.335 & & SSZ & 10 (19.2) & $6(31.6)$ & $4(12.1)$ & 0.090 \\
\hline & $\begin{array}{l}\text { SLE and other } \\
\text { CTD'S }\end{array}$ & $11(21.2)$ & $3(15.8)$ & $8(24.2)$ & 0.364 & & Azathioprine & $2(3.8)$ & $2(10.5)$ & $0(0)$ & \\
\hline & Others & $6(11.5)$ & $3(15.8)$ & $3(9.1)$ & 0.380 & & Iguratimod & $3(5.8)$ & $1(5.3)$ & $2(6.1)$ & 0.129 \\
\hline \multirow{10}{*}{$\begin{array}{l}\text { Comorbidities } \\
\text {-n (\%) }\end{array}$} & DM2 & $12(23.1)$ & $9(47.4)$ & 3.(9.1) & 0.003 & & Combination & $13(25.0)$ & $6(31.6)$ & $7(21.2)$ & 0.701 \\
\hline & & & & & & DMARDS(B) & Adalimumab & $1(20.0)$ & $0(0)$ & $1(25.0)$ & 0.659 \\
\hline & HTN & $13(25)$ & $8(42.1)$ & $5(15.2)$ & 0.035 & $-n(\%)$ & Etanercept & $1(20.0)$ & $0(0)$ & $1(25.0)$ & \\
\hline & & & & & & & Rituximab & $3(60.0)$ & $1(100.0)$ & $2(50.0)$ & \\
\hline & CKD & $2(3.8)$ & $2(10.5)$ & $0(0)$ & 0.129 & CO RADS & 0 & $11(21.2)$ & $0(0)$ & 11 (33.3) & $<0.001$ \\
\hline & & & & & & -n (\%) & 1 & $9(17.3)$ & $0(0)$ & $9(27.3)$ & \\
\hline & Hypothyroidism & $12(23.1)$ & $4(21.1)$ & $8(24.2)$ & 0.537 & & 2 & $3(5.8)$ & $0(0)$ & $3(9.1)$ & \\
\hline & & & & & & & 3 & $3(5.8)$ & $2(10.5)$ & $1(3)$ & \\
\hline & Multimorbidity & $8(15.4)$ & $6(31.6)$ & $2(6.1)$ & 0.021 & & 4 & $11(21.2)$ & $7(36.8)$ & $4(12.1)$ & \\
\hline & & & & & & & 5 & $15(28.8)$ & $10(52.6)$ & $5(15.2)$ & \\
\hline
\end{tabular}

Disclosure of Interests: None declared

DOI: 10.1136/annrheumdis-2021-eular.3137

\section{\begin{tabular}{|l|l}
\hline POS1236 IMPACT OF COVID-19 ON INITIATION AND RENEWAL \\
\hline
\end{tabular} OF BIOTHERAPIES AND TARGETED SYNTHETIC TREATMENTS}

$\underline{\text { P. Richette }}^{1}$, M. Allez $z^{2}$, V. Descamps ${ }^{3}$, L. Perra ${ }^{4}$, S. Pilet ${ }^{5}$, M. Maravic ${ }^{6}$. ${ }^{1}$ Lariboisiere Hospital, Rheumatology, France, France; ${ }^{2}$ St Louis Hospital, Gastroenterology, Paris, France; ${ }^{3}$ Bichat Hospital, Dermatology, Paris, France; ${ }^{4} I Q V I A$, Ai \& Data engine, Courbevoie, France; ${ }^{5}$ QQVIA, Analytic solutions, Courbevoie, France; ${ }^{6}$ IQVIA, Real World Solutions, Courbevoie, France

Background: During the epidemic's peak of COVID-19, scientific societies published recommendations on biotherapy and targeted synthetic treatment (B/TST) use in patients with chronic articular inflammatory diseases, inflammatory bowel diseases, and psoriasis.

Objectives: The objective was to evaluate the impact of COVID-19 in France on initiation and renewal of B/TST.

Methods: LRx contains all anonymized medication dispenses prescribed in outpatient care in a representative panel of French retails pharmacies, including data of near 40 million patients. The impact of B/TST initiation and renewal were studied using 2019 as reference and dispense deliveries data of pharmacies with regular flew in order to perform the comparison. B/TST considered were abatacept, anti-TNF, anti-IL6, anti-IL17, anti-IL12/23 or anti-IL23, JAK inhibitors (JAKi) and other classes such as aprelimast, aminosalicylates (AS), hydroxychloroquine (HCQ), and methotrexate (MTX). A treatment initiated was defined as a treatment not delivered in the past 12 months, and conversely for a treatment renewal. Results were presented as raw one and expressed in percentage of patients having at least one B/TST delivery in each therapeutic classes of interest in 2020 compared to 2019 used as reference year (period from week 12 to week 19 considered and corresponding to the lockdown period in France).

Results: During the lockdown period, a decrease in initiation was observed for patients treated with: abatacept (405 in 2019 vs 227 in 2020: $-44 \%, p<0.001$ ), anti-TNF (1156 vs 1058, $-31 \%, p<0.001$ ), anti-IL17 (415 vs 206, $-50 \%, p<0.001$ ), anti-IL12-23 (395 vs 339, -12\%, $p=0.16)$, JAKi (289 vs $174,-39 \%, p=0.006)$, contrasting with an increase for Tociliumab (117 vs $445,+152 \%, p=0,01)$. We found a decrease of $7 \%$ (2171 vs 2015, $p=0,35$ ), $44 \%$ (405 vs 227, $p<0.001$ ), 30\% (3430 vs $2390, p \mathrm{p}<0.001$ ) of AS, aprelimast and MTX initiation, respectively, and an increase of $173 \%$ (1708 vs $4671, p=0.11$ ) of $H C Q$ initiation. No decrease for the renewal of B/TST was observed

Conclusion: During the epidemic's peak, initiation of AS, MTX, biotherapies (except for tocilizumab), and JAKi dramatically decreased without impacting their renewal. Two treatments were mainly initiated, tocilizumab probably due to a switch from intravenous to subcutaneous injection and HCQ in relation to its presumably effect on COVID-19. Overall, recommendations from scientific societies have been followed.

Disclosure of Interests: None declared

DOI: 10.1136/annrheumdis-2021-eular.3146

\section{POS1237}

ALGORITHM IDENTIFYING CHRONIC INFLAMMATORY DISEASES TREATED BY BIOTHERAPY AND/OR TARGETED SYNTHETIC TREATMENTS IN OUTPATIENT CARE IN FRANCE: FEASIBILITY, PRELIMINARY RESULTS, AND IMPACT OF COVID-19

P. Richette ${ }^{1}$, M. Allez ${ }^{2}$, V. Descamps ${ }^{3}$, L. Perra ${ }^{4}$, S. Pilet ${ }^{5}$, M. Maravic ${ }^{6}$

${ }^{1}$ Lariboisiere Hospital, Rheumatology, Paris, France; ${ }^{2}$ St Louis Hospital,

Gastroenterology, Paris, France; ${ }^{3}$ Bichat Hospital, Dermatology, Paris, France;

${ }^{4} I Q V I A, A i$ \& Data engine, Courbevoie, France; ${ }^{5}$ QVVIA, Analytic Solutions,

Courbevoie, France; ${ }^{6} I Q V I A$, Real World Solutions, Courbevoie, France

Background: Discriminating chronic inflammatory diseases under biotherapy and/or targeted synthetic treatments (B/TST) using medico-administrative databases are challenging but required for medico-economic analyses focusing on these diseases.

Objectives: The objective was to evaluate the feasibility of using a medication dispense data in order to identify patients with chronic inflammatory diseases under B/TST in outpatient care setting and evaluate the impact of COVID-19 in France

Methods: LRx contains all anonymized medication dispenses prescribed in outpatient care in a representative panel of French retails pharmacies, including data of near 40 million patients. Patients having at least one B/TST delivered in 2019 were selected. An algorithm was constructed using different steps, including a machine learning step by transfer learning applied in patient classified as having a rheumatologic condition in order to differentiate rheumatoid arthritis (RA) from spondyloarthritis (SA). We numbered 190,640 patients, of which $87.8 \%$ were classified in one of the following diseases: RA, SA, psoriatic arthritis (PA), psoriasis, inflammatory bowel diseases (IBD) (extrapolated data to France). Descriptive analysis was performed. The impact of COVID-19 on biotherapy initiation was evaluated during the lockdown period (W2-W19 2020) in these different conditions.

Results: Among the 167,468 patients under B/TST, 20.7\%, 18.4\%, $6.5 \%, 37.9 \%$ and $16.5 \%$ were considered as having a psoriasis, IBD, PA, RA, and SA, respectively. Female patients were more frequent in RA and PA (> 60\%); younger (< 20 years) and older patients (74 years) were found in patients with IBD $(5.7 \%)$ and RA (12.6\%), respectively. Contrasting with IBD, SA, and RA patients were mainly under anti-TNF treatment (> 90\% for IBD and SA, $73.5 \%$ for RA), psoriasis and PA patients received a range of broadly well-balanced of B/TST. Among the immunosuppressant, methotrexate was mostly prescribed in RA $(58.4 \%)$, PA $(34.1 \%)$, and psoriasis (14.1\%), and azathioprine in IBD (19.9\%). Oral corticosteroid delivered at least 4 times in 2019 were mostly found to be associated with a RA condition $(28 \%)$. A significant decrease of biotherapy initiation was observed during the lockdown in France in patients with IBD $(-20 \%, \mathrm{p}=0.03)$ and psoriasis $(-54 \%, p<0.0001)$, not significant decrease in patients with SA $(-6 \%)$ and increase in RA patients $(+23 \%)$.

Conclusion: The algorithm was able to identify patients with chronic inflammatory diseases under B/TST delivered in outpatient care and will allow to follow-up its management and study the COVID-19 impact on biotherapy initiation. An external validation needs to be performed

Disclosure of Interests: None declared

DOI: 10.1136/annrheumdis-2021-eular.3165 